\title{
HDA6 is required for jasmonate response, senescence and flowering in Arabidopsis
}

\author{
Keqiang $\mathrm{Wu}^{1, *}$, Lin Zhang ${ }^{2}$, Changhe $\mathrm{Zhou}^{2}$, Chun-Wei $\mathrm{Yu}^{1}$ and Vijaya Chaikam ${ }^{2}$ \\ ${ }^{1}$ Institute of Plant Biology, College of Life Science, National Taiwan University, Taipei 10617, Taiwan \\ ${ }^{2}$ Department of Biology, West Virginia University, Morgantown, WV 26506-6057, USA
}

Received 3 September 2007; Revised 3 November 2007; Accepted 5 November 2007

\begin{abstract}
Post-translational modifications of histones, including acetylation, play a key role in modulating dynamic changes in chromatin structure and gene activity. Histone acetylation is modulated through the action of histone acetyltransferases and deacetylases. HDA6 is a RPD3-type histone deacetylase in Arabidopsis. The Arabidopsis HDA6 mutant, axe1-5, and HDA6 RNAinterfering (HDA6-RNAi) plants displayed higher levels of acetylated $\mathrm{H} 3$ compared with wild-type, suggesting that HDA6 affects histone acetylation levels globally. The expression of the jasmonate responsive genes, PDF1.2, VSP2, JIN1, and ERF1, was down-regulated in axe1-5 and HDA6-RNAi plants. Furthermore, axe1-5 and HDA6-RNAi plants displayed increased leaf longevity compared with the wild type. The expression of the senescence-associated genes, SAG12 and SEN4, was down-regulated in the axe1-5 and HDA6-RNAi plants. In addition, axe1-5 and HDA6-RNAi plants displayed late-flowering. The expression of $F L C$ was upregulated and hyperacetylated in axe 1-5 and HDA6-RNAi plants, suggesting that HDA6 is required to deacetylate $F L C$ chromatin and thereby repress its expression. Our results suggest that HDA6 is involved in jasmonate response, senescence, and flowering in Arabidopsis.
\end{abstract}

Key words: HDA6, flowering, histone deacetylases, jasmonic acid, senescence.

\section{Introduction}

In higher organisms, many different patterns of gene expression are required for proper development to occur and to allow for specific responses to environmental cues.
Transcriptional regulation of gene expression plays a fundamental role in plant response to environmental stimuli. A fundamental mechanism controlling gene expression is the ability of many transcription factors to access the genome of eukaryotes. Post-translational modifications of histones, including acetylation, methylation, phosphorylation, and ubiquitinylation play a key role in modulating dynamic changes in chromatin structure and gene activity (Berger, 2002; Reyes et al., 2002). Histone acetylation levels are determined by the action of histone acetyltransferases and histone deacetylases (HDACs). Plant HDACs can be grouped into four different classes, namely, RPD3class, HDA1-class, SIR2-class, and HD2-class (Pandey et al., 2002). RPD3-class HDACs are the homologues of the yeast protein, RPD3 (Taunton et al., 1996). Four RPD3-class HDACs, HDA19 (also called AtRPD3A or AtHD1), HDA6 (AtRPD3B), HDA7, and HDA9 (Wu et al., 2000; Murfett et al., 2001; Tian and Chen, 2001; Pandey et al., 2002) were identified in Arabidopsis. Mutations in HDA6 affected transgene expression, DNA methylation, and regulation of rRNA genes (Murfett et al., 2001; Aufsatz et al., 2002; Probst et al., 2004; Earley et al., 2006). Antisense suppression and T-DNA disruption of HDA19 expression resulted in a range of developmental abnormalities including apical defect, reduced fertility, delayed flowering and altered light response (Wu et al., 2000; Tian and Chen, 2001; Tian et al., 2003; Benhamed et al., 2006; Long et al., 2006). More recently, it was found that HDA18, a HDA1-class HDAC, is required for normal cellular patterning of the Arabidopsis root epidermis (Xu et al., 2005).

There is increasing evidence indicating that histone acetylation is involved in the plant response to abiotic and biotic stresses (Stockinger et al., 2001; Devoto et al., 2002; Jang et al., 2003; Kim et al., 2004; Song et al.,

* To whom correspondence should be addressed. E-mail: kewu@ntu.edu.tw 
2005; Zhou et al., 2005). It was suggested that histone acetyltransferases may be recruited through transcription factors, such as CBF1, to cold-induced genes, through multiprotein complexes similar to those found in other eukaryotes (Stockinger et al., 2001). Recently, it was demonstrated that HDA6 can interact with COI1, an F-box protein, that is involved in jasmonate (JA)-mediated plant defence responses, suggesting a possible role for HDA6 in plant-pathogen interaction (Devoto et al., 2002). Our recent studies indicate that the expression of Arabidopsis HDA19 and HDA6 can be induced by ethylene and JA (Zhou et al., 2005). In addition, overexpression of HDA19 in Arabidopsis induced ethylene- and JA-regulated $P R$ gene expression and resulted in increased resistance to the pathogen Alternaria brassicicola. These studies provide evidence that RPD3-type HDACs, HDA19, and HDA6, may play an important role in ethylene- and JA-signalling and pathogen responses. A recent study by Song et al. (2005) provides direct evidences that HDACs are involved in ABA and abiotic stress responses. It was found that an AP2/EREBP transcription repressor, AtERF7, interacts with the Arabidopsis thaliana homologue of a human global corepressor of transcription, AtSin3, which, in turn, may interact with a HDAC.

In the present study, it is reported that HDA6 is required for the JA response, senescence, and flowering in Arabidopsis. The expression of the JA-responsive genes, PDF1.2, VSP2, JIN1, and ERF1, was down-regulated in the Arabidopsis HDA6 mutant, axe1-5, and HDA6-RNA interfering (HDA6-RNAi) plants. Furthermore, axe1-5 and HDA6-RNAi plants displayed increased leaf longevity compared with the wild type. The expression of the senescence-associated genes, SAG12 and SEN4, was also down-regulated in the axel-5 and HDA6-RNAi plants. In addition, axe 1-5 and HDA6-RNAi plants displayed lateflowering. The expression of $F L C$ was up-regulated and hyperacetylated in axe1-5 and HDA6-RNAi plants, suggesting that HDA6 is required to deacetylate $F L C$ chromatin and thereby repress its expression.

\section{Materials and methods}

\section{Plant materials}

Arabidopsis thaliana was grown in a growth chamber under longday (LD, $16 \mathrm{~h}$ light and $8 \mathrm{~h}$ dark) or short-day (SD, $8 \mathrm{~h}$ light and 16 $\mathrm{h}$ dark) conditions after a $2-4 \mathrm{~d}$ stratification period. For growth under sterile conditions, seeds were surface-sterilized $[15 \mathrm{~min}$ incubation in $5 \%(\mathrm{v} / \mathrm{v})$ sodium hypochlorite, and rinsed three times in sterile distilled water] and sown on half-strength Murashige and Skoog (MS) salts (Sigma, St Louis, MO) supplemented with $1 \%$ sucrose, $\mathrm{pH} 5.7$, and $0.8 \%(\mathrm{w} / \mathrm{v})$ agar in Petri dishes.

\section{Plasmid construction}

To generate the HDA6:GUS construct, a $1.3 \mathrm{~kb}$ promoter for HDA6 was PCR amplified by using the primer pairs, $5^{\prime}$-TCCAGATCTGCAGTTGTAGG-3' and 5'-GCCTCCATCTCCGTCTCTCACTCAGAATC- $3^{\prime}$. The resulting PCR product was then digested by
PstI and NcoI and subcloned into the pCAMBIA1381 binary vector (Cambia, Canberra, Australia). To generate 35S:HDA6-GFP, the HDA6 coding region was PCR amplified using the primer pairs, 5' -AATTTCCCGGGCATGAGGCAGACGAAAGCGGCA-3' and 5' -AATTGAGCTCTTAAGACGATGGAGGATTCACG-3', to replace $A t H D 2 A$ in the AtHD2A-GFP construct (Zhou et al., 2004). DNA and protein sequence analysis was carried out using BLAST searches (http://www.ncbi.nlm.nih.gov/BLAST/) and the Vector NTI Suite program (InforMax Inc., Bethesda, MD).

\section{Plant transformation and selection}

Plant transformation plasmids were electroporated into Agrobacterium tumefaciens GV3101 as described by Shaw (1995). The Agrobacterium-mediated transformation of Arabidopsis thaliana was performed as described by Clough and Bent (1998). $\mathrm{T}_{1}$ seeds were harvested, dried at $25{ }^{\circ} \mathrm{C}$, and germinated on sterile medium containing $40 \mu \mathrm{g} \mathrm{ml}^{-1}$ hygromycin to select the transformants. Surviving $T_{1}$ plantlets were transferred to soil to set seeds $\left(T_{2}\right)$.

\section{$\beta$-glucuronidase assays and GFP localization}

For histochemical GUS assay, Arabidopsis tissues were incubated in a $0.5 \mathrm{mg} \mathrm{ml}^{-1}$ solution of 5-bromo-4-chloro-indolyl $\beta$-D-glucuronide (X-Glu) in $100 \mathrm{mM}$ sodium phosphate buffer, $\mathrm{pH}$ 7.0, and incubated at $37{ }^{\circ} \mathrm{C}$ overnight, followed by washing with $70 \%$ ethanol to remove the chlorophyll (Jefferson, 1988).

For GFP localization, transgenic seeds were germinated on a MS medium. Protoplasts were isolated from transgenic Arabidopsis seedlings as described by Weigel and Glazebrook (2002). The fluorescence photographs of protoplasts were taken using an Olympus florescent microscope (Tokyo, Japan) fitted with fluorescein isothiocyanate filters (excitation filter, $450-490 \mathrm{~nm}$; emission filter, $520 \mathrm{~nm}$; and dichroic mirror, $510 \mathrm{~nm}$ ).

\section{Semi-quantitative RT-PCR analysis}

One microgram of total RNA was used for the first-strand cDNA synthesis after incubation at $65{ }^{\circ} \mathrm{C}$ for $10 \mathrm{~min}$. cDNA was synthesized in a volume of $20 \mu \mathrm{l}$ that contained MoMLV reverse transcriptase buffer (Promega, Madison, Wisconsin, USA), $10 \mathrm{mM}$ dithiothreitol, $1.5 \mu \mathrm{M}$ poly(dT) primer, $0.5 \mathrm{mM}$ dNTPs, $2 \mathrm{U}$ of MoMLV reverse transcriptase at $37{ }^{\circ} \mathrm{C}$ for $1 \mathrm{~h}$. All PCR reactions were performed with $0.5 \mathrm{U}$ of Taq polymerase (PGC Scientific, Gaithersburg, Maryland, USA), the buffer provided by the supplier, $0.2 \mathrm{mM}$ dNTPs, and a pair of primers $(0.1 \mu \mathrm{M}$ each $)$ in a final volume of $20 \mu \mathrm{l}$. PCR parameters differed for each gene: thermocycling conditions were $94{ }^{\circ} \mathrm{C}$ for 2 min followed by $25-35$ cycles of $94{ }^{\circ} \mathrm{C}$ for $1 \mathrm{~min}, 55-68{ }^{\circ} \mathrm{C}$ for $1 \mathrm{~min}$, and $72{ }^{\circ} \mathrm{C}$ for $2 \mathrm{~min}$, with a final polymerization step at $72{ }^{\circ} \mathrm{C}$ for $10 \mathrm{~min}$. The genespecific primer pairs used for the RT-PCR are listed in the Table 1.

\section{Protein gel blot analysis}

Nuclear proteins were isolated as described by Weigel and Glazebrook (2002). $500 \mathrm{mg}$ of Arabidopsis seedling tissues were homogenized in $1 \mathrm{ml}$ of Honda buffer $(2.5 \%$ Ficoll $400,5 \%$ dextran T40, 0.4 M sucrose, $25 \mathrm{mM}$ TRIS-HCl [pH 7.4], $10 \mathrm{mM} \mathrm{MgCl}_{2}$, $10 \mathrm{mM} \beta$-mercaptoethenol, $100 \mu \mathrm{g} \mathrm{ml}^{-1}$ phenylmethylsulphonyl fluoride, $0.5 \mu \mathrm{g} \mathrm{ml}^{-1}$ antipain, $0.5 \mu \mathrm{g} \mathrm{ml}^{-1}$ leupeptin) and filtered through a $62 \mu \mathrm{m}$ nylon mesh. $0.5 \%$ Triton X-100 was then added to the extract, which was incubated for $15 \mathrm{~min}$ on ice and centrifuged at $1500 \mathrm{~g}$ for $5 \mathrm{~min}$. The pellet was washed with Honda buffer containing $0.1 \%$ Triton $\mathrm{X}-100$, gently resuspended in $1 \mathrm{ml}$ of Honda buffer, and centrifuged at $100 \mathrm{~g}$ for $5 \mathrm{~min}$ to pellet starch and cell debris. The supernatant was transferred to a microcentrifuge tube and centrifuged at $1800 \mathrm{~g}$ for 5 min to pellet the nuclear.

The nuclear extract was suspended in $200 \mu$ l of $5 \times$ SDS-PAGE loading buffer [0.2 M TRIS-HCl (pH 6.8), 25\% SDS, 25\% glycerol 
Table 1. Primers used for RT-PCR

\begin{tabular}{|c|c|}
\hline Genes & Primers \\
\hline HDA19 & 5'-ACAAGATGCCGGAGCATGAA-3' and 5'-TTTAGGAGGAAACGCCTGCT-3' \\
\hline HDA6 & 5'-TAGAGCCGGACAACAAACTC-3' and 5' - TTCACGTCTGGCTCTGGGTT-3' \\
\hline HDA7 & 5'-GGTGATCCGTTTGGTACATT-3' and 5'-TCTTCTCCATGTCCACTTCC-3'; \\
\hline HDA9 & $5^{\prime}$-TTACAGGAGGTGGAGGATAC-3' and 5' -CGTTATCGTTGTCTCCATCG-3' \\
\hline VSP2 & 5'-TTCTATGCCAAAGGACTTGC-3' and 5'-GAGTGGATTTGGGAGCTTAA-3' \\
\hline ERF1 & 5'-GTTCTCTTTGCTGCTTTCGAC-3' and 5'-TTCTCCGTCTCATCGAGTGT-3' \\
\hline PDF1.2 & 5'-ATGCTGGAACTACCACTACC-3' and 5'-CCATGTTTGGCTCCTTCAAG-3' \\
\hline JIN1 & $5^{\prime}$-TCGGTGACGCAATCGCTTAC- $3^{\prime}$ and 5'-CTTGCTCTGAGCTGTTCTTG-3' \\
\hline SAG12 & 5'-CAGCTGCGGATGTTGTTG-3' and 5'-CCACTTTCTCCCCATTTTG-3' \\
\hline SEN4 & 5'-TCTTCTTCACGACTCTTCTC-3 and 5'-TTGCCCAATCGTCTGCGTTC-3 \\
\hline RPS17 & 5'-ATGATAACGTCGTCCCTAAC-3' and 5'-GCTGAGACTCCAAGGGAAGG-3' \\
\hline FLC & 5'-TTAGTATCTCCGGCGACTTGAACCCA-3' and 5' -AGATTCTCAACAAGCTTCAACATGAG-3' \\
\hline MAF1 & 5'- GCTAGGAAGGCAGAACTGAT -3' and 5'-CCAATCCGTACATTCAGACAC-3' \\
\hline MAF2 & 5'-TGTCCGTAACTAGAGCTAGG -3' and 5'-CCTTAATTTCCACATTGGCGC-3' \\
\hline MAF4 & 5'-ATTAGGTCAGAAGAATTAGTCGGAGAAAAC-3' and 5'-CTTGGATGACTTTTCCGTAGCAGGGGGAAG-3' \\
\hline MAF5 & 5'-GGGGATTAGATGTGTCGGAAGAGTGAAG-3' and 5'-GATCCTGTCTTCCAAGGTAACACAAAGG-3' \\
\hline UBIQUITIN & $5^{\prime}$-GATCTTTGCCGGAAAACAATTGGAGGATGGT-3' and 5'-CGACTTGTCATTAGAAAGAAAGAGATAACAGG-3' \\
\hline
\end{tabular}

and $12.5 \% 2$-mercaptoethanol]. The protein samples were loaded on $15 \%$ polyacrylamide gel and blotted onto a nitrocellulose membrane. The membrane was blocked in PBS containing 3\% dry milk for $60 \mathrm{~min}$ and then incubated with $0.01-0.05 \mu \mathrm{g} \mathrm{ml} \mathrm{m}^{-1}$ of antiacetyl-histone H3 K9 and K14 antibody (Catalogue no. 06-599, Upstate, Charlottesville, VA) for $2 \mathrm{~h}$ at room temperature. After washing, the primary antibody was detected with secondary antirabbit horseradish peroxidase coupled antibody (Amersham, Buckinghamshire, England) at room temperature for $45 \mathrm{~min}$. Visualization was achieved using the ECL system (Amersham).

\section{Chromatin immunoprecipitation analysis}

Chromatin immunoprecipitation (ChIP) analysis was carried out as described previously (Johnson et al., 2002; Gendrel et al., 2005). Chromatin extracts were prepared from young leaves treated by formaldehyde. The chromatin was sheared to an average length of $500-1500$ bp by sonication and immunoprecipitated with the antiacetyl-histone H3 K9 and K14 antibody (Catalogue no. 06-599, Upstate, Charlottesville, VA). The immunocomplexes were harvested with Protein A agarose and heated at $65^{\circ} \mathrm{C}$ for $5 \mathrm{~h}$ to release DNA cross-linked to the immunoprecipitated proteins. The DNA cross-linked to the immunoprecipitated proteins was analysed by PCR. To assess non-specific binding, the immunoprecipitation reaction was also performed in the absence of antibody. The primers used to amplify FLC were FLC-B (5'-TGTAGGCACGACTTTGGTAACACC-3' and 5' - GCAGAAAGAACCTCCACTCTACATC-3'), FLC-P (5' -CGACTTGAACCCAAACCTGAGGATCAAAT- $3^{\prime}$ and 5' $^{\prime}$-AGAAGATAAAAGGGGGAACAAATGAAAAC-3'), FLC-CH (5' -CTGCGACCATGATAGATACATGAGA-3' and $5^{\prime}$ - TTCACTCAACAACATCGAGCACG-3'), FLC-V2 (5' - ATAGATTTGCCTCATATTTATGTGATTGT-3' and 5' ${ }^{\prime}$-TTCATTATAGATCCGTACCAAAGAGGTTG-3'), and FLC-U (5'-GCTGATAAGGGCGAGCGTTTG- $3^{\prime}$ and $5^{\prime}$-AAGCCGTAGGCTTCTTCACTG-3'). The primer pair JP1595 (5' -CGTTTCGCTTTCCTTAGTGTTAGCT-3') and JP1596 (5'-AGCGAACGGATCTAGAGACTCACCTTG- $3^{\prime}$ ) was used to amplify ACTIN (Johnson et al., 2002). 30 cycles of PCR $\left(94{ }^{\circ} \mathrm{C}\right.$ for $25 \mathrm{~s}, 59^{\circ} \mathrm{C}$ for $35 \mathrm{~s}, 72{ }^{\circ} \mathrm{C}$ for $\left.30 \mathrm{~s}\right)$ were performed as separate reactions for $F L C$ and ACTIN amplification.

\section{Measurement of chlorophyll content and photochemical efficiency}

From about 15 DAE (days after leaf emergence) onwards, the sixth rosette leaf, which was fully grown, was chosen for chlorophyll extraction and photochemical efficiency of photosystem II (PSII) measurement. Chlorophyll was extracted from equal volumes of leaf discs by grinding the leaves in liquid nitrogen and dissolving in $80 \%$ acetone. Chlorophyll concentration per g fresh weight of leaf was calculated as described by Porra et al. (1989). The photochemical efficiency of PSII was deduced from the characteristics of chlorophyll fluorescence (Oh et al., 1997) using a portable plant efficiency analyser (Hansatech Instruments, Morfolk, England). The ratio of maximum variable fluorescence to maximum yield of fluorescence $\left(F_{\mathrm{v}} / F_{\mathrm{m}}\right)$, which corresponds to the potential quantum yield of the photochemical reactions of PSII, was used as the measurement of the photochemical efficiency of PSII (Oh et al., 1997).

\section{Results}

\section{Expression and localization of HDA6}

The expression pattern of $H D A 6: G U S$ was analysed by fusing the $1.3 \mathrm{~kb} H D A 6$ promoter fragment to a $G U S$ reporter gene in Arabidopsis plants. In 2-week-old transgenic seedlings, GUS was strongly expressed in cotyledons and hypocotyls (Fig. 1A). In adult plants, GUS expression was detected in the mature leaves and stems. GUS activity was also detected in all parts of flowers except the stamens. However, GUS activity was not detected in the seeds (Fig. 1A). The expression pattern of HDA6:GUS is therefore different from that of HDA19: $G U S$, which was expressed in all of the organs analysed (Zhou et al., 2005). Expression of HDA6 and other RPD3type $H D A$ s was analysed further by using RT-PCR. As shown in Fig. 1B, HDA6 and HDA19 transcripts were detected in roots, leaves, stems, and flowers of Arabidopsis. By comparison, HDA9 transcript could only be detected in flowers.

To investigate the cellular distribution of HDA6 protein, an in vivo targeting experiment was performed using green fluorescent protein (GFP). HDA6-GFP gene fusion driven by the $35 \mathrm{~S}$ promoter was created and introduced into Arabidopsis using the Agrobacterium-mediated floral 
dip method (Clough and Bent, 1998). To confirm that the fusion protein entered the nucleus, the fluorescence of GFP was monitored at the cellular level. Protoplasts were isolated from seedlings of transgenic Arabidopsis, and localization of the fusion protein was determined by a fluorescence microscope. As shown in Fig. 1C, bright green fluorescence was observed only in the nuclei of HDA6-GFP transgenic plants. This indicates that HDA6GFP fusion protein was localized in the nucleus of the
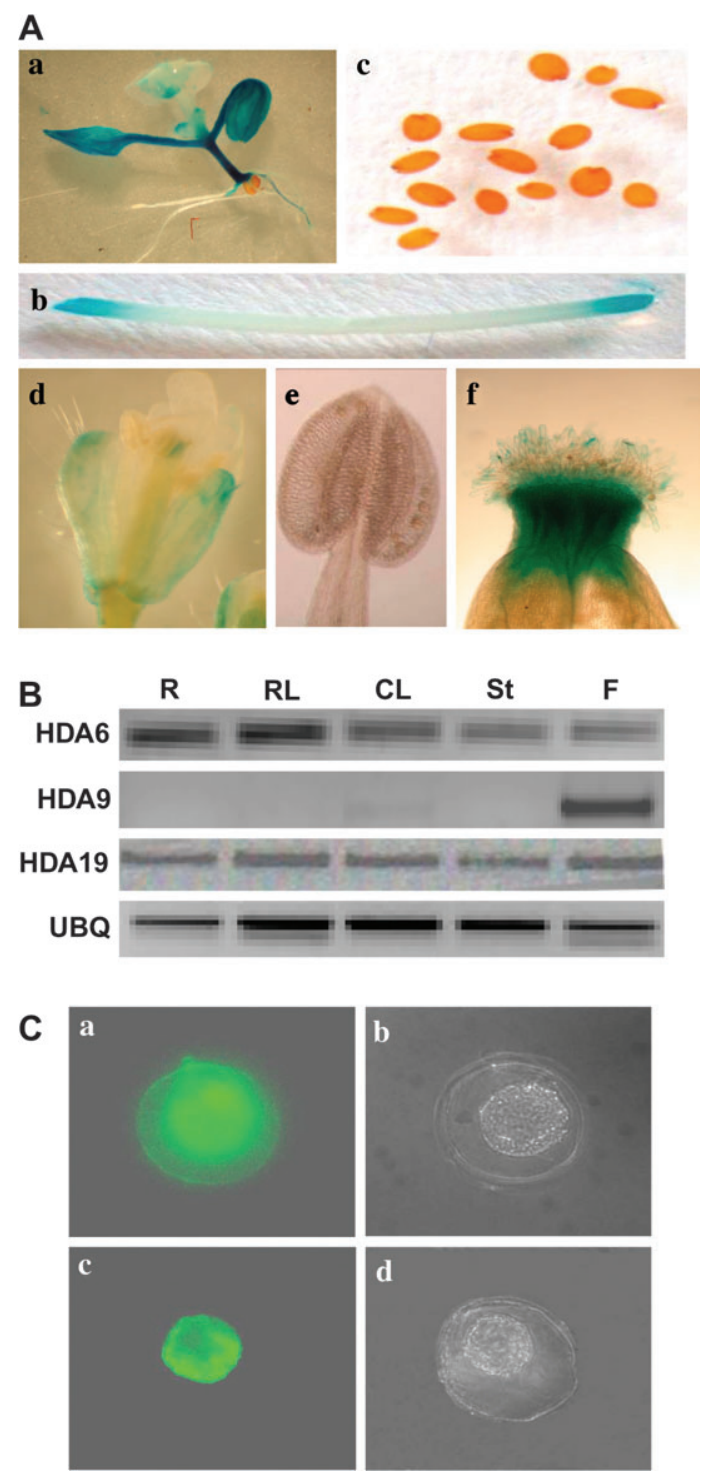

Fig. 1. HDA6 expression and HDA6 localization. (A) GUS staining in the HDA6:GUS plants from a seedling (a), stem (b), seeds (c), flower (d), stamen (e), and stigma (f). (B) RT-PCR analysis of expression of HDA6, HDA9, and HDA19 genes in Arabidopsis. Total RNA for RTPCR analysis was isolated from roots $(\mathrm{R})$, rosette leaves $(\mathrm{RL})$, cauline leaves $(\mathrm{CL})$, stems $(\mathrm{St})$, and flowers $(\mathrm{F})$ of A. thaliana. Ubiquitin $(U B Q)$ was shown as an internal control. (C) Subcellular localization of HDA6GFP. Protoplasts were isolated from the leaves of $35 S: G F P(a, b)$ and 35S:HDA6-GFP (c, d) transgenic Arabidopsis plants. GFP fluorescence was examined by fluorescence microscopy under UV light $(a, c)$ and white light $(b, d)$.
Arabidopsis cells. This result is consistent with the observation of Earley et al. (2006), who demonstrated the nuclear localization of HDA6-Flag by immunolocalization. These results support the idea that HDA6 is involved in transcription regulation.

\section{HDA6 affected JA-responsive gene expression}

To investigate the function of HDA6 further, two HDA6RNAi lines, CS24038 and CS24039 (ecotype Wassilewskija [Ws]), generated by expressing a transgene that encodes double-stranded HDA6 RNA (Plant Chromatin Database: http://chromdb.org) were analysed. RT-PCR analysis indicated that HDA6 transcript levels in the HDA6-RNAi plants were significantly reduced relative to non-transgenic control plants (Fig. 2A). CS24039 plants had greater reduction in HDA6 expression compared with CS24038 plants. The expression of other RPD3-class HDACs, HDA19 and HDA9, was not affected in the HDA6-RNAi plants.

The levels of acetylated histone $\mathrm{H} 3$ in HDA6-RNAi plants were analysed by protein gel blot analysis. As shown in Fig. 2B, there were obvious increased levels of acetylated $\mathrm{H} 3$ in two HDA6-RNAi lines compared with the Ws wild type, suggesting that the HDA6 transcript level affects histone acetylation levels globally. Similarly, increased level of acetylated $\mathrm{H} 3$ was also observed in the HDA6 mutant, axe1-5 [ecotype Columbia (Col)], when compared with the Col wild type (Fig. 2B). axel-5 is a splice site mutant that has a base change at an intron splice site resulting in two HDA6 transcripts with altered lengths (Murfett et al., 2001). Changed levels of acetylated H3 in

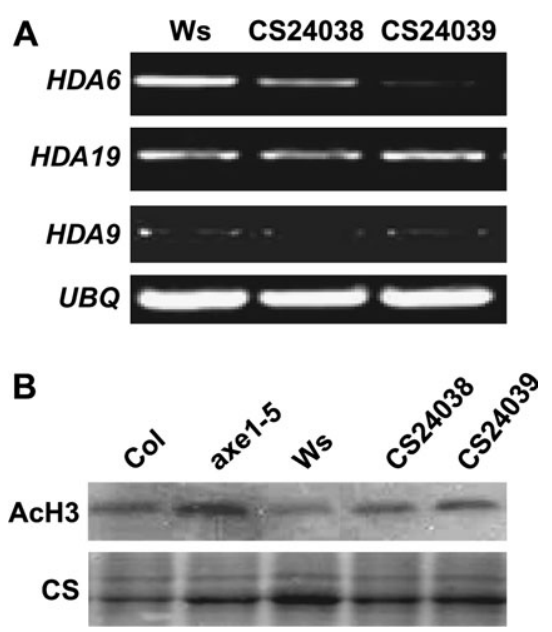

Fig. 2. Expression of HDA6 and levels of acetylated $\mathrm{H} 3$ in HDA6RNAi lines. (A) RT-PCR analysis of HDA6, HDA19, and HDA9 expression. Total RNA for RT-PCR analysis was isolated from leaf tissues of Ws wild-type and HDA6-RNAi lines (CS24038 and CS24039). Ubiquitin (UBQ) was shown as an internal control. (B) Protein gel blot analysis detecting acetylated H3 (AcH3) (top panel) using $\alpha$-AcH3 antibodies on protein extracts from Col wild-type, axel-5, Ws wild-type, and HDA6-RNAi lines (CS24038 and CS24039). Bottom panel, Coomassie staining (CS) shows equal protein loading. 
axe1-5 and HDA6-RNAi plants support the observation that HDA6 has a HDAC activity (Earley et al., 2006).

The interaction of HDA6 with COI1, an F-box protein that was required for JA-mediated plant defence responses, has been demonstrated (Devoto et al, 2002), suggesting a possible role of HDA6 in JA response. To investigate further the involvement of HDA6 in the JA-responsive pathway, semiquantitative RT-PCR was conducted to examine transcript levels of JA-responsive genes, PDF1.2, VSP2, JIN1, and ERF1 (Lorenzo et al., 2003, 2004), in the axel-5 and HDA6-RNAi plants. As shown in Fig. 3A, expression of $P D F 1.2$, VSP2, JIN1, and ERF1 was down-regulated in axe1-5 and HDA6-RNAi plants. Expression of PDF1.2, VSP2, JIN1, and ERF1 can be induced by JA in Col wildtype plants (Fig. 3B). By comparison, expression of these JA-responsive genes can not be induced by JA in axel-5 plants (Fig. 3B). Down-regulation of PDF1.2, VSP2, JIN1, and ERF1 in axel-5 and HDA6-RNAi plants suggests that HDA6 is required for expression of the JA-responsive genes.

\section{axe1-5 and HDA6-RNAi plants displayed increased leaf longevity}

JA was found to be involved in leaf senescence in Arabidopsis (He et al., 2002). To investigate whether
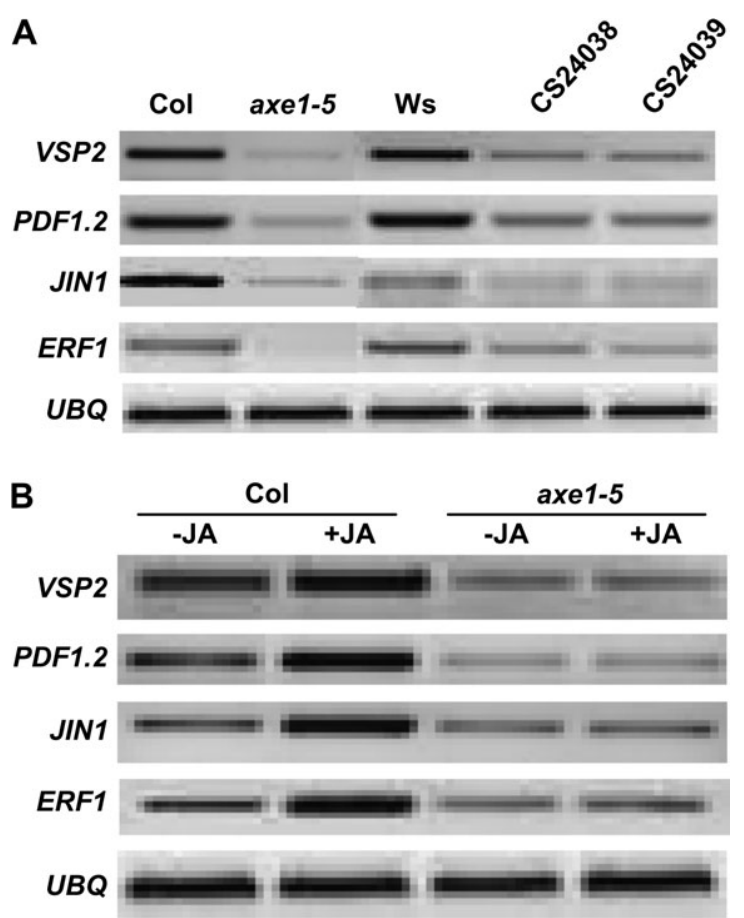

Fig. 3. JA-responsive gene expression in axel-5 and HDA6-RNAi plants. (A) Expression of PDF1.2, VSP2, JIN1, and ERF1 in Col wildtype, axel-5, Ws wild-type, and HDA6-RNAi lines (CS24038 and CS24039) plants. Total RNA for RT-PCR analysis was isolated from leaf tissues. Ubiquitin $(U B Q)$ was shown as an internal control. (B) Induction of VSP2, PDF1.2, JIN1, and ERF1 expression by JA in Col wild-type and axel-5 plants. Total RNA for RT-PCR analysis was isolated from leaf tissues with $(+)$ or without $(-) 0.1 \mathrm{mM} \mathrm{JA}$ treatment for $12 \mathrm{~h}$. Ubiquitin $(U B Q)$ was shown as an internal control.
HDA6 is required for leaf senescence, leaf longevity of the axe1-5 and HDA6-RNAi plants was examined. The phenotype of individual leaves grown under the long-day (LD, $16 \mathrm{~h}$ light and $8 \mathrm{~h}$ dark) condition was followed from the formation of a visually recognizable leaf primordium ( $1 \mathrm{~mm}$ in size) and the leaf was considered dead when the entire leaf turned yellow (Grbi and Bleecker, 1995). As shown in Fig. 4A, the leaves of axe1-5 and HDA6-RNAi plants turned yellow much slower and showed increased leaf longevity when compared with their wild-type counterparts.

Leaf longevity was also assessed by measuring typical senescence-associated physiological markers, such as chlorophyll contents and photochemical efficiency of PSII (Fan et al., 1997; Oh et al., 1997). Chlorophyll contents decline at the onset of senescence and it is considered as an important indicator of the rate of senescence (Nam, 1997). Chlorophyll contents were measured from $15 \mathrm{DAE}$ (days after leaf emergence), which was also the day of the 6th rosette leaf that was fully grown. At $45 \mathrm{DAE}$, the leaves of Ws and Col wild type lost $65-75 \%$ of their chlorophylls, whereas these of axe1-5 and HDA6-RNAi just lost 15-35\% (Fig. 4B). Delayed senescence of the mutants was also defined as delay in the decrease in photosynthetic activity (Fig. 4C). The PSII efficiency has also been shown to be an effective indicator of leaf senescence in plants (Lu and Zhang, 1998a, b). It was demonstrated that, during senescence, the PSII efficiency declines rapidly, leading to a loss of photosynthetic capabilities of the leaves and leading to the eventual death of the leaves. This analysis indicated that axe1-5 and HDA6-RNAi leaves consistently showed later development of senescence-associated changes. These results suggest that decreased expression of HDA6 causes increased leaf longevity in Arabidopsis.

Leaf senescence is accompanied by the decreased expression of genes related to photosynthesis and protein synthesis genes (PAGs) (Bate et al., 1991) and increased expression of senescence-associated genes (SAGs) (Nam, 1997). The expression patterns were examined of two $S A G$ genes, SAG12 and SEN4, which have been shown to be up-regulated during senescence (Gan and Amasino, 1997; Park et al, 1998), in the axel-5 and HDA6-RNAi plants. As shown in Fig. 5, the expression of SAG12 and SEN4 was down-regulated in the axel-5 and HDA6-RNAi plants when compared with the wild-type. By comparison, the expression of RPS17, a PAG gene that encodes the chloroplast ribosomal protein S17 (Woo et al., 2002), was up-regulated in axe1-5 and HDA6-RNAi plants (Fig. 5). These results support the idea that HDA6 is required for $S A G$ s expression and therefore it is involved in senescence progression. In the absence of $H D A 6, P A G$ genes are upregulated, which leads to a higher rate of photosynthesis, resulting in higher PSII efficiencies and higher chlorophyll contents in axe 1-5 and HDA6-RNAi plants. 

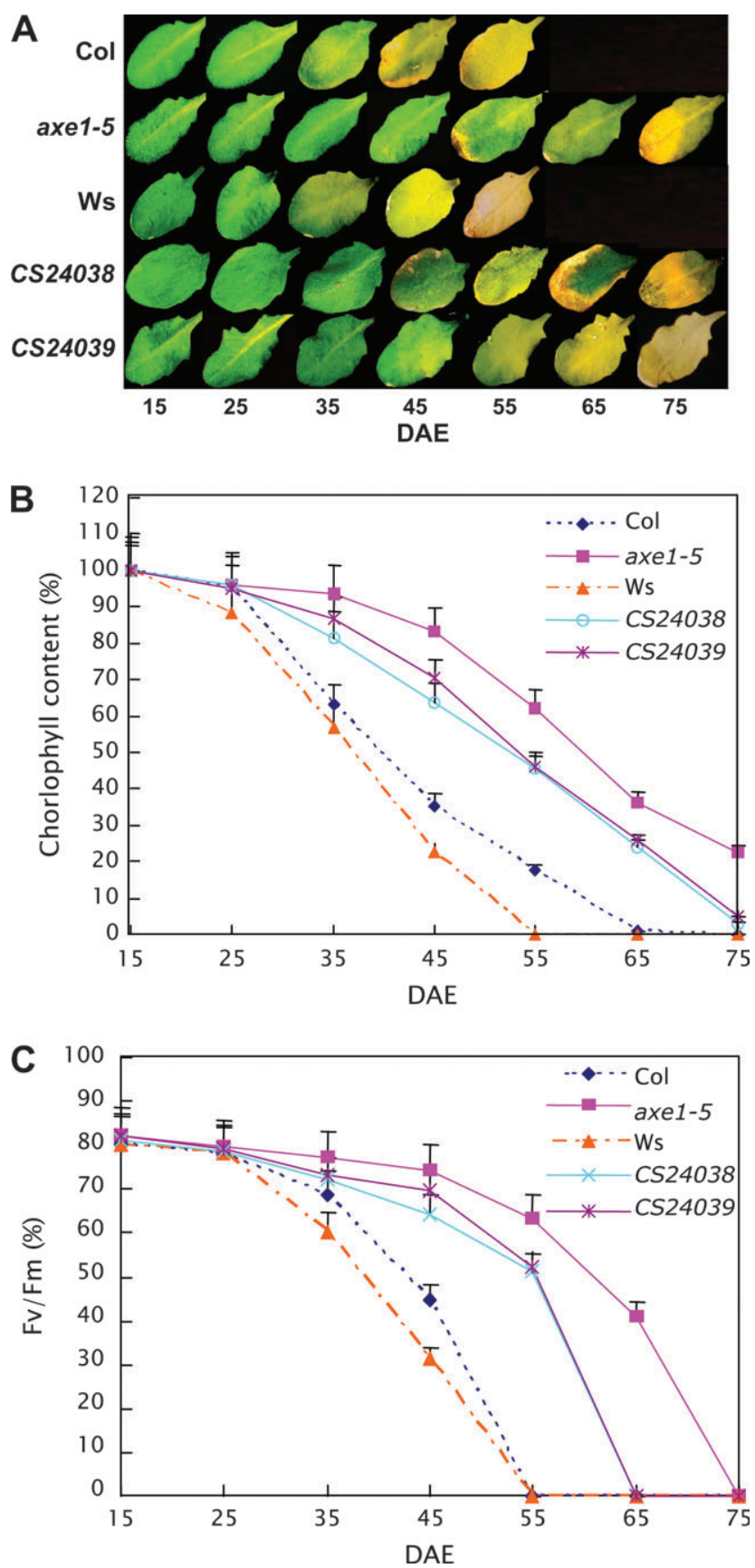

Fig. 4. Age-dependent senescence symptoms in axel-5 and HAD6RNAi plants. (A) The age-dependent senescence phenotype of Col wild-type (Col), axel-5, Ws wild-type (Ws), and HAD6-RNAi (CS24038 and CS24039) leaves grown under the long-day (LD, $16 \mathrm{~h}$ light and $8 \mathrm{~h}$ dark) conditions. Photographs show representative leaves at each time point. Pictures were taken every $10 \mathrm{~d}$ from $15 \mathrm{DAE}$ (days after leaf emergence). (B, C) Chlorophyll content (B) and photochemical efficiency of PSII (C). Data were collected every $10 \mathrm{~d}$ from 15 DAE, when the 6th rosette leaves were just fully grown. $F_{\mathrm{v}} / F_{\mathrm{m}}$, maximum quantum yield of PSII electron transport (maximum variable fluorescence/maximum yield of fluorescence). Error bars indicate SE $(n \geqslant 15)$.

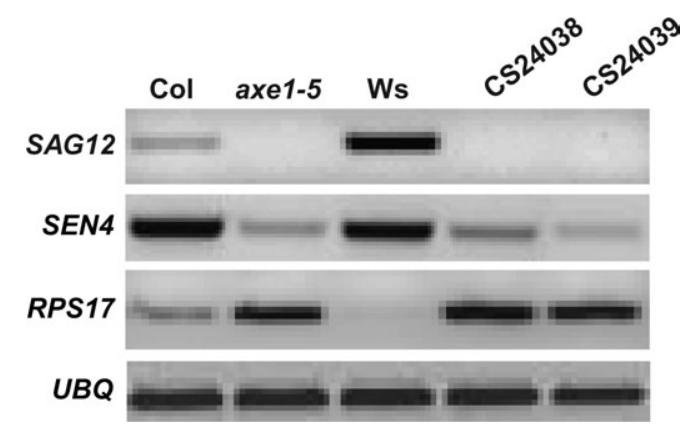

Fig. 5. Expression of SAG12, SEN4, and RPS17 in axe1-5 and HDA6RNAi plants. RT-PCR analysis of SAG12, SEN4, and RPS17 expression in Col wild-type (Col), axel-5, Ws wild-type (Ws), and HDA6-RNAi (CS24038 and CS24039) plants. Total RNA for RT-PCR analysis was isolated from leaf tissues. Ubiquitin $(U B Q)$ was shown as an internal control.

\section{axe1-5 and HDA6-RNAi plants displyed delayed flowering}

axe1-5 and HDA6-RNAi mutants displayed later flowering phenotypes, as measured by the days to bolting and the rosette leaf numbers at flowering (Fig. 6). Col, Ws, and HDA6 mutants were grown in long-day (LD, $16 \mathrm{~h}$ light and $8 \mathrm{~h}$ dark) and short-day (SD, $8 \mathrm{~h}$ light and $16 \mathrm{~h}$ dark) conditions, and the flowering time was compared. The flowering of axe1-5 and HDA6-RNAi plants was greatly delayed in SD as well as in LD in terms of both the days to flowering and the rosette leaf numbers at flowering initiation (Fig. 6). axe1-5 plants did not flower even at $104 \mathrm{~d}$ after germination in SD, when some rosette leaves showed senescence. The delay in flowering time of axe 1-5 and HDA6-RNAi plants was completely corrected by $45 \mathrm{~d}$ of vernalization at $4{ }^{\circ} \mathrm{C}$ (Fig. 6B). These observations suggest that HDA6 is involved in the autonomous pathway of flowering.

The delayed flowering of the axe1-5 and HDA6-RNAi plants prompted us to analyse whether the expression of $F L C$, a transcription factor that controls the transition from vegetative to reproductive development, was affected. RT-PCR analysis indicated that expression of $F L C$ was increased in axel-5 and HDA6-RNAi plants compared with the wild type (Fig. 7). In addition to FLC, other MADS-box transcriptional factors, such as MAF1, MAF2, MAF4, and MAF5, are closely related to FLC and also function as repressors of flowering in Arabidopsis (Scortecci et al., 2001; Ratcliffe et al., 2003). It was found that expression of MAF4 and MAF5 was also increased in axe 1-5 and HDA6-RNAi plants (Fig. 7).

Chromatin immunoprecipitation (ChIP) analysis was carried out using the anti-acetyl-histone $\mathrm{H} 3$ antibody to determine if the acetylation level of $F L C$ was affected in HDA6 mutants. A 295 base pair region (FLC-CH) of the first intron of $F L C$ was shown to be hyperacetylated in the $f l d$ and fve mutants of the autonomous flowering pathway (He et al., 2003). Therefore the histone acetylation status 

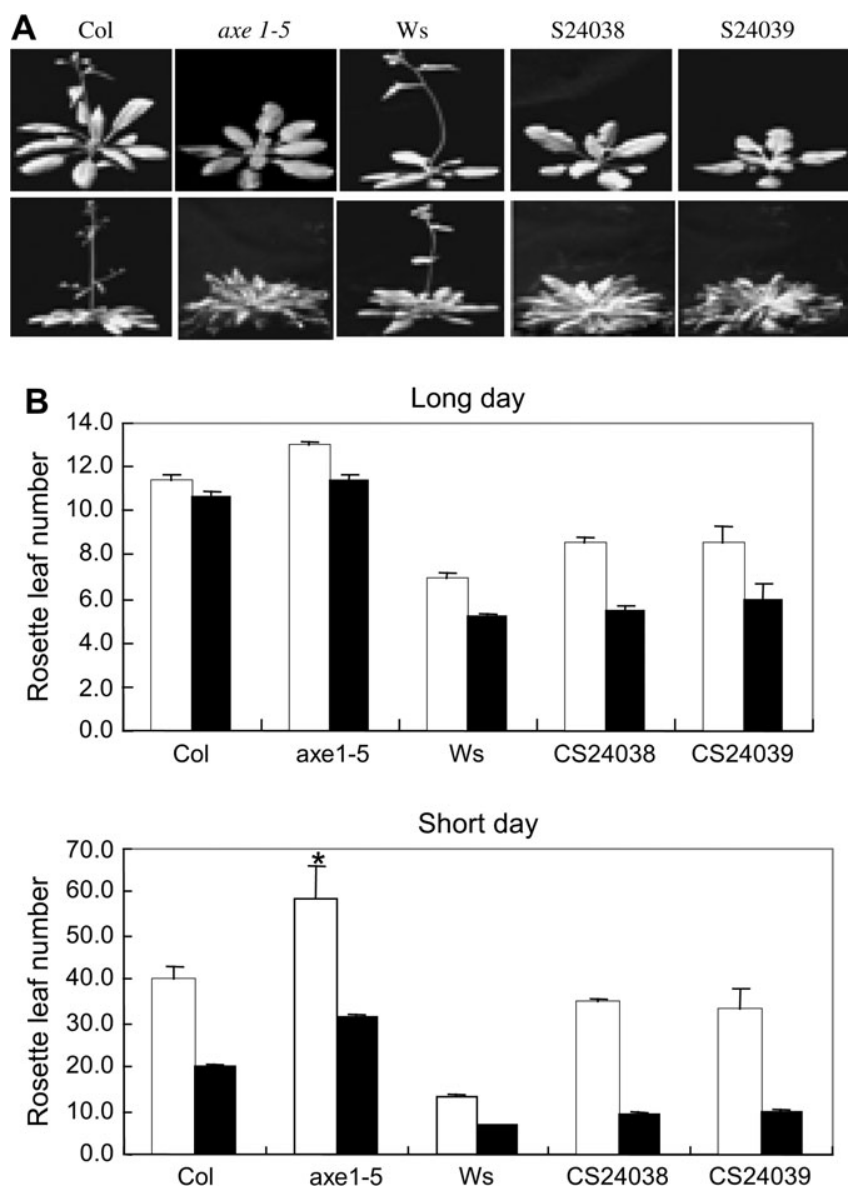

Fig. 6. Delayed flowering of axe1-5 and HDA6-RNAi plants. (A) Plants were grown under LD (top panel) or SD (bottom panel) without vernalization. Col wild-type and axe1-5 plants were grown under LD and SD for $31 \mathrm{~d}$ and $87 \mathrm{~d}$, respectively; whereas Ws wild-type and HDA6-RNAi (CS24038 and CS24039) plants were grown under LD and SD for $24 \mathrm{~d}$ and $64 \mathrm{~d}$, respectively. (B) Rosette leaf number $\pm \mathrm{SE}$ at bolting of Col, axe 1-5, Ws, and two HDA6-RNAi lines (CS24038 and CS24039) grown under LD or SD conditions without vernalization (white columns) or after vernalization for $45 \mathrm{~d}$ (black columns). Values are the mean of 10-20 plants per genotype. (Asterisk) Under SD without vernalization, axe 1-5 plants did not flower $104 \mathrm{~d}$ after germination and the rosette leaf number of axe1-5 was counted at $104 \mathrm{~d}$.

of this region was analysed in $F L C$. As shown in Fig. 8B, $F L C$ displayed hyperacetylation of histone $\mathrm{H} 3$ in the axe1-5 and HDA6-RNAi plants compared with the wild type, suggesting that HDA6 is required to deacetylate $F L C$ chromatin and repress $F L C$ expression. The histone acetylation status of other regions of $F L C$ was also analysed in the axe1-5 mutant. There was increased acetylation of histone $\mathrm{H} 3$ in the 1st exon and 1st intron of $F L C$, but not in the promoter and the $3^{\prime}$ UTR regions (Fig. 8C).

\section{Discussion}

The HDA6 mutant, axel, was isolated based on deregulated expression of auxin-responsive transgenes (Murfett

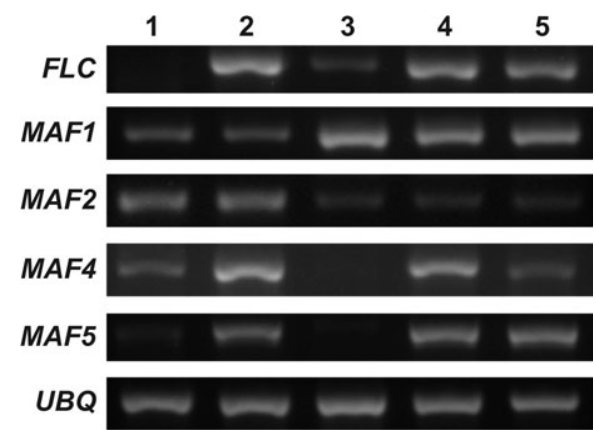

Fig. 7. Expression of $F L C$ in axe1-5 and HDA6-RNAi plants. RT-PCR analysis of FLC, MAF1, MAF2, MAF4, and MAF5 expression in Col (1), axel-5 (2), Ws (3), CS24038 (4), and CS24039 (5) plants. Total RNA for RT-PCR analysis was isolated from leaf tissues. Ubiquitin $(U B Q)$ was shown as an internal control.

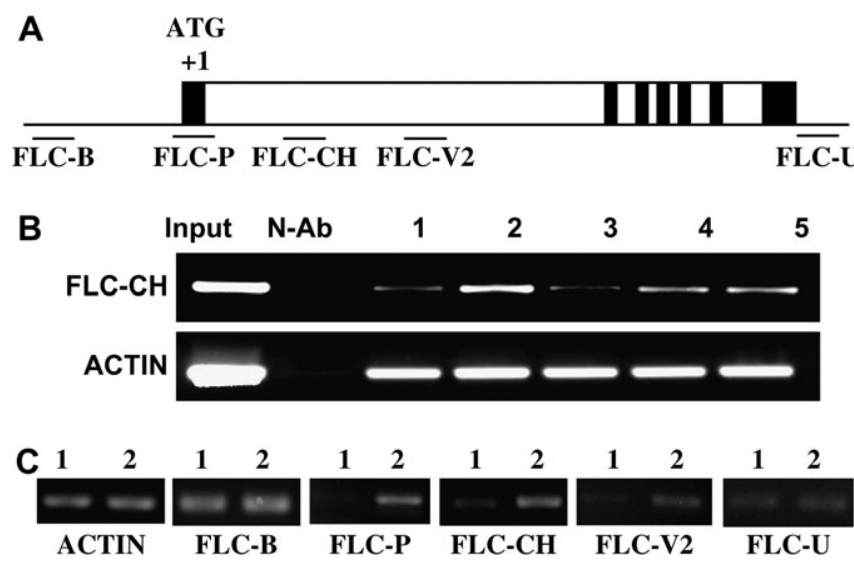

Fig. 8. Acetylation status of $F L C$ in axe1-5 and HDA6-RNAi plants. (A) Relative positions of amplified $F L C$ regions used in the ChIP assay. The black boxes correspond to exons, white boxes to introns and single lines to $5^{\prime}$ or $3^{\prime}$ UTR. (B) ChIP analyses of the acetylation status of histone $\mathrm{H} 3$ in $F L C$ chromatin in the Col wild-type (1), axel-5 (2), Ws (3), CS24038 (4), and CS24039 (5) plants. The input is Col chromatin before immunoprecipitation; $\mathrm{N}-\mathrm{Ab}$, control sample lacking antibody; ACTIN served as an internal control of the ChIP analysis. (C) ChIP analyses of the acetylation status of histone $\mathrm{H} 3$ in $F L C$ chromatin in the Col wild-type (1) and axe1-5 (2) plants. Five regions were examined in FLC chromatin. ACTIN served as an internal control of the ChIP analysis.

et al., 2001). Since then, other mutant alleles of HDA6, rtsl and sill, were also isolated based on their effects on specific transgene expression (Aufsatz et al., 2002; Probst et al., 2004). It was shown that mutations in HDA6 result in loss of transcriptional silencing from several repetitive transgenic and endogenous templates. In the axe1-5 mutant, significant hyperacetylation is identified in the nucleolus organizer regions that contain the rDNA repeats (Probst et al., 2004). It was suggested that HDA6 might play a role in regulating activity of rRNA genes (Earley et al., 2006), and this control might be functionally linked to silencing of other repetitive templates and to its role in RNA-directed DNA methylation (Probst et al., 2004).

This study indicates that HDA6 is involved in JA response, senescence, and flowering. It has been observed 
that the JA-responsive genes, PDF1.2, JIN1, ERF1, and VSP2, were down-regulated in axe1-5 and HDA6-RNAi plants, suggesting that HDA6 is required for the JAresponsive pathway. The finding that HDA6 expression can be induced by JA also supports a role for HDA6 in the JA-response pathway (Zhou et al., 2005). Our previous studies indicate that HDA19 plays an important role in the JA-signalling pathway (Zhou et al., 2005). It would be interesting to know whether HDA6 and HDA19 have redundant function in the JA-response pathway. Further study by analysing HDA6 and HDA19 double mutants will be useful to address this question. HDA6 mutants displayed delayed leaf senescence compared with wildtype plants. The delayed leaf senescence in axel-5 and HDA6-RNAi plants was demonstrated by measuring changes in typical senescence-associated physiological markers such as chlorophyll content and photochemical efficiency (Fan et al., 1997; Oh et al., 1997). Expression of two SAG genes, SAG12 and SEN4, was decreased in axe1-5 and HDA6-RNAi plants when compared with the wild type. By comparison, expression of a $P A G$ gene, RPS17, was up-regulated in axe1-5 and HDA6-RNAi plants. These results suggest that HDA6 is required for $S A G \mathrm{~s}$ expression and is involved in senescence progression.

Although histone deacetylases have often been associated with the repression of gene expression, recent studies in yeast and animal cells indicate that histone deacetylation can also be required as a transcriptional activation signal. Deletion of yeast histone deacetylases Rpd3, or Hda1 results in decreased transcription of a number of genes and an increase rather than a loss of silencing, pointing to an alternative role of HDACs as transcriptional activators (Rundlett et al., 1996; Bernstein et al., 2000). In addition, SIN3, a major component of the HDACcontaining transcriptional repressor complex, can function as both a transcriptional corepressor and a transcriptional coactivator (Nawaz et al., 1994). Furthermore, Mouse Histone Deacetylase 1 (HDAC1) can act as a negative regulator as well as a positive regulator of transcription (Zupkovitz et al., 2006). These studies indicate that gene regulation by acetylation is more dynamic and HDACs may also function as activators (Nusinzon and Horvath, 2005). An alternative explanation for the repression of gene activity in axe1-5 and HDA6 RNAi plants could be that HDA6 may target transcription repressors.

The interaction of HDA6 with COI1, an F-box protein that was required for JA-mediated plant defence responses, has been demonstrated (Devoto et al., 2002). However, the function and biological significance of this interaction is unknown. F-box proteins interact with SKP1 and cullin proteins to form E3 ubiquitin ligases known as the SCF complexes that selectively recruit regulatory proteins targeted for ubiquitination (Vierstra, 2003). Coimmunoprecipitation experiments confirmed the interaction of COI1 with SKP1-like proteins and HDA6 in planta. Regulation of HDAC activities by ubiquitination has been demonstrated in mammalian cells (Gaughan et al., 2005). It was proposed that COI1 may form a functional E3-type ubiquitin ligase in plants to regulate expression of JA-responsive genes, possibly by targeted ubiquitination of a histone deacetylase (Devoto and Turner, 2003). In addition to its role in targeting proteins for proteolytic degradation by the proteasome, ubiquitination can also regulate protein location, activity, and interaction with binding partners (Schnell and Hicke, 2003; Caron et al., 2005). Further analysis is required to reveal whether HDA6 is regulated by ubiquitination and the biological function of this modification.

Plant flowering is regulated by both environmental and endogenous cues. Molecular genetic studies on the mechanism of flowering in Arabidopsis, a quantitative long-day plant, have revealed four major flowering pathways: the photoperiod, autonomous, vernalization, and gibberellin pathways (Mouradov et al., 2002; Boss et al., 2004; Henderson and Dean, 2004). A central player in the autonomous and vernalization flowering pathways is FLC, which blocks flowering by inhibiting genes required to switch the meristem from vegetative to flower development. axe1-5 and HDA6-RNAi plants flowered later than the wild-type plants in both long-day and short-day photoperiods and flowered rapidly after exposure to a prolonged period of cold (vernalization), which is characteristic of autonomous-pathway mutants (He et al., 2003). The genes in the autonomous pathway, such as FVE and FLD, promote flowering by suppressing FLC. More recent studies indicated that histone acetylation is involved in plant flowering (He et al., 2003; Ausin et al., 2004; Kim et al., 2004). Mutations in FLD and FVE, two proteins that were found in histone deacetylase complexes, result in hyperacetylation of histones in FLC chromatin, up-regulation of $F L C$ expression, and delayed flowering, indicating that the autonomous pathway regulates flowering in part by histone deacetylation (He et al., 2003; Ausin et al., 2004; Kim et al., 2004). It was found that FLC was up-regulated and hyperacetylated in the axe1-5 and HDA6-RNAi plants, suggesting that HDA6 is required to deacetylate $F L C$ chromatin and repress its expression. FVE and FLD may therefore act with HDA6 to repress $F L C$ expression.

In summary, our studies indicate that, in addition to its role in transgene expression, DNA methylation, and regulating activity of rRNA genes (Murfett et al., 2001; Aufsatz et al., 2002; Probst et al., 2004; Earley et al., 2006), HDA6 is involved in many aspects of plant development. In particular, HDA6 is required for JA response, senescence, and flowering in Arabidopsis. The role for JA in leaf senescence in Arabidopsis was demonstrated based on the fact that exogenous application of JA induces leaf senescence, and this induction requires an intact JA signalling pathway (He et al., 2002). In 
addition, it was shown that the endogenous JA level in senescing leaves increased to nearly $500 \%$ of that in nonsenescent counterpart leaves. Thus HDA6 may be involved in leaf senescence via the JA-signalling pathway. Arabidopsis mutants that affect both senescence and flowering time were isolated previously (Barth et al., 2006). For example, the Arabidopsis ascorbic aciddeficient mutant vtcl exhibits a delayed senescence and flowering phenotype (Pavet et al., 2005). By comparison, mutation of the matrix metalloproteinase At2-MMP that is important for the degradation and remodelling of the extracellular matrix causes late flowering but early senescence in Arabidopsis (Golldack et al., 2002). These results suggest that flowering and senescence could be regulated independently. It remains to be determined whether HDA6 is independently involved in these processes. Analysis of the genetic interaction between the HDA6 mutation and mutations affecting JA signalling, senescence or flowering is required to dissect further the role of HDA6 in these pathways.

\section{Acknowledgements}

We thank Brian Miki for critical reading of the manuscript. We are grateful to Tom Guilfoyle for providing axel-5 seeds, and Chromatin Functional Genomics Consortium and Arabidopsis Biological Resource Center for providing HDA6-RNAi seeds. This work was supported by grants from the National Science Council of Taiwan (NSC 95-2321-B-002-026 and 96-2311-B-002-013-MY2).

\section{References}

Aufsatz W, Mette MF, Van Der Winden J, Matzke M, Matzke AJ. 2002. HDA6, a putative histone deacetylase needed to enhance DNA methylation induced by double-stranded RNA. EMBO Journal 21, 6832-6841.

Ausin I, Alonso-Blanco C, Jarillo JA, Ruiz-Garcia L, MartinezZapater JM. 2004. Regulation of flowering time by FVE, a retinoblastoma-associated protein. Nature Genetics 36, 162-166.

Barth C, De Tullio M, Conklin PL. 2006. The role of ascorbic acid in the control of flowering time and the onset of senescence. Journal of Experimental Botany 57, 1657-1665.

Bate NJ, Rothstein SJ, Thompson JE. 1991. Expression of nuclear and chloroplast photosynthesis-specific genes during leaf senescence. Journal of Experimental Botany 42, 801-811.

Benhamed M, Bertrand C, Servet C, Zhou DX. 2006. Arabidopsis GCN5, HD1, and TAF1/HAF2 interact to regulate histone acetylation required for light-responsive gene expression. The Plant Cell 18, 2893-2903.

Berger SL. 2002. Histone modification in transcriptional regulation. Current Opinion in Genetic Development 12, 142-148.

Bernstein BE, Tong JK, Schreiber SL. 2000. Genomewide studies of histone deacetylase function in yeast. Proceedings of the National Academy of Sciences, USA 97, 13708-13713.

Boss PK, Bastow RM, Mylne JS, Dean C. 2004. Multiple pathways in the decision to flower: enabling, promoting, and resetting. The Plant Cell 16, S18-S31.

Caron C, Boyault C, Khochbin S. 2005. Regulatory cross-talk between lysine acetylation and ubiquitination: role in the control of protein stability. Bioessays 27, 408-415.
Clough SJ, Bent AF. 1998. Floral dip: a simplified method for Agrobacterium-mediated transformation of Arabidopsis thaliana. The Plant Journal 16, 735-743.

Devoto A, Nieto-Rostro M, Xie D, Ellis C, Harmston R, Patrick E, Davis J, Sherratt L, Coleman M, Turner JG. 2002. COI1 links jasmonate signalling and fertility to the SCF ubiquitin-ligase complex in Arabidopsis. The Plant Journal 32, 457-466.

Devoto A, Turner JG. 2003. Regulation of jasmonate-mediated plant responses in Arabidopsis. Annals of Botany 92, 329-337.

Earley K, Lawrence RJ, Pontes O, Reuther R, Enciso AJ, Silva M, Neves N, Gross M, Viegas W, Pikaard CS. 2006. Erasure of histone acetylation by Arabidopsis HDA6 mediates large-scale gene silencing in nucleolar dominance. Genes and Development 20, 1283-1293.

Fan L, Zheng S, Wang X. 1997. Antisense suppression of phospholipase $\mathrm{D} \alpha$ retards abscisic acid- and ethylene-promoted senescence of postharvest Arabidopsis leaves. The Plant Cell 9, 2183-2196.

Gan S, Amasino RM. 1997. Making sense of senescence. Plant Physiology 113, 313-319.

Gaughan L, Logan IR, Neal DE, Robson CN. 2005. Regulation of androgen receptor and histone deacetylase 1 by Mdm2mediated ubiquitylation. Nucleic Acids Research 33, 13-26.

Gendrel AV, Lippman Z, Martienssen R, Colot V. 2005. Profiling histone modification patterns in plants using genomic tiling microarrays. Nature Methods 2, 213-218.

Golldack D, Popova OV, Dietz KJ. 2002. Mutation of the matrix metalloproteinase At2-MMP inhibits growth and causes late flowering and early senescence in Arabidopsis. Journal of Biological Chemistry 277, 5541-5547.

Grbi V, Bleecker AB. 1995. Ethylene regulates the timing of leaf senescence in Arabidopsis. The Plant Journal 8, 595-602.

He Y, Fukushige H, Hildebrand DF, Gan S. 2002. Evidence supporting a role of jasmonic acid in Arabidopsis leaf senescence. Plant Physiology 128, 876-884.

He Y, Michaels SD, Amasino RM. 2003. Regulation of flowering time by histone acetylation in Arabidopsis. Science 302, 1751-1754.

Henderson IR, Dean C. 2004. Control of Arabidopsis flowering: the chill before the bloom. Development 131, 3829-3838.

Jang IC, Pahk YM, Song SI, Kwon HJ, Nahm BH, Kim JK. 2003. Structure and expression of the rice class-I type histone deacetylase genes OsHDAC1-3: OsHDAC1 overexpression in transgenic plants leads to increased growth rate and altered architecture. The Plant Journal 33, 531-541.

Jefferson R. 1988. Plant reporter genes: the GUS gene fusion system. In: Setlow JK, ed. Genetic engineering: principles and methods, Vol. 10. New York: Plenum Press, 247-263.

Johnson L, Cao X, Jacobsen S. 2002. Interplay between two epigenetic marks: DNA methylation and histone H3 lysine 9 methylation. Current Biology 12, 1360-1367.

Kim HJ, Hyun Y, Park JY, et al. 2004. A genetic link between cold responses and flowering time through FVE in Arabidopsis thaliana. Nature Genetics 36, 167-171.

Long JA, Ohno C, Smith ZR, Meyerowitz EM. 2006. TOPLESS regulates apical embryonic fate in Arabidopsis. Science 312, $1520-1523$.

Lorenzo O, Piqueras R, Sanchez-Serrano JJ, Solano R. 2003. ETHYLENE RESPONSE FACTOR1 integrates signals from ethylene and jasmonate pathways in plant defence. The Plant Cell 15, 165-178.

Lorenzo O, Chico JM, Sanchez-Serrano JJ, Solano R. 2004. JASMONATE-INSENSITIVE1 encodes a MYC transcription factor essential to discriminate between different jasmonateregulated defence responses in Arabidopsis. The Plant Cell 16, 1938-1950. 
Lu CM, Zhang J. 1998a. Modifications in photosystem II photochemistry in senescent leaves of maize plants. Journal of Experimental Botany 49, 1671-1679.

Lu CM, Zhang J. 1998b. Changes in photosystem II function during senescence of wheat leaves. Physiologia Plantarum 104, 239-247.

Mouradov A, Cremer F, Coupland G. 2002. Control of flowering time: interacting pathways as a basis for diversity. The Plant Cell 14, S111-S130.

Murfett J, Wang X, Hagen G, Guilfoyle TJ. 2001. Identification of Arabidopsis histone deacetylase HDA6 mutants that affect transgene expression. The Plant Cell 13, 1047-1061.

Nam HG. 1997. The molecular genetic analysis of leaf senescence. Current Opinion in Biotechnology 8, 200-207.

Nawaz Z, Baniahmad C, Burris TP, Stillman DJ, O'Malley BW, Tsai MJ. 1994. The yeast SIN3 gene product negatively regulates the activity of the human progesterone receptor and positively regulates the activities of GAL4 and the HAP1 activator. Molecular and General Genetics 245, 724-733.

Nusinzon I, Horvath CM. 2005. Histone deacetylases as transcriptional activators? Role reversal in inducible gene regulation. Science STKE 2005, re11.

Oh SA, Park JH, Lee GI, Paek KH, Park SK, Nam HG. 1997. Identification of three genetic loci controlling leaf senescence in Arabidopsis thaliana. The Plant Journal 12, 527-535.

Pandey R, Muller A, Napoli CA, Selinger DA, Pikaard CS, Richards EJ, Bender J, Mount DW, Jorgensen RA. 2002. Analysis of histone acetyltransferase and histone deacetylase families of Arabidopsis thaliana suggests functional diversification of chromatin modification among multicellular eukaryotes. Nucleic Acids Research 30, 5036-5055.

Park JH, Oh SA, Kim YH, Woo HR, Nam HG. 1998. Differential expression of senescence-associated mRNAs during leaf senescence induced by different senescence-inducing factors in Arabidopsis. Plant Molecular Biology 37, 445-454.

Pavet V, Olmos E, Kiddle G, Mowla S, Kumar S, Antoniw J, Alvarez ME, Foyer CH. 2005. Ascorbic acid deficiency activates cell death and disease resistance responses in Arabidopsis. Plant Physiology 139, 1291-1303.

Porra RJ, Thompson WA, Kriedemann PE. 1989. Determination of accurate extinction coefficients and simultaneous equations for assaying chlorophylls $a$ and $b$ extracted with four different solvents: verification of the concentration of chlorophyll standards by atomic absorption spectroscopy. Biochimica et Biophysica Acta 975, 384-394.

Probst AV, Fagard M, Proux F, et al. 2004. Arabidopsis histone deacetylase HDA6 is required for maintenance of transcriptional gene silencing and determines nuclear organization of rDNA repeats. The Plant Cell 16, 1021-1034.

Ratcliffe OJ, Kumimoto RW, Wong BJ, Riechmann JL. 2003. Analysis of the Arabidopsis MADS AFFECTING FLOWERING gene family: MAF2 prevents vernalization by short periods of cold. The Plant Cell 15, 1159-1169.

Reyes JC, Hennig L, Gruissem W. 2002. Chromatin-remodeling and memory factors: new regulators of plant development. Plant Physiology 130, 1090-1101.

Rundlett SE, Carmen AA, Kobayashi R, Bavykin S, Turner BM, Grunstein M. 1996. HDA1 and RPD3 are members of distinct yeast histone deacetylase complexes that regulate silencing and transcription. Proceedings of the National Academy of Sciences, USA 93, 14503-14508.

Schnell JD, Hicke L. 2003. Non-traditional functions of ubiquitin and ubiquitin-binding proteins. Journal of Biological Chemistry 278, 35857-35860.

Scortecci KC, Michaels SD, Amas.ino RM. 2001. Identification of a MADS-box gene, FLOWERING LOCUS M, that represses flowering. The Plant Journal 26, 229-236.

Shaw CH. 1995. Introduction of cloning plasmids into Agrobacterium tumefaciens. Methods in Molecular Biology 49, 33-37.

Song CP, Agarwal M, Ohta M, Guo Y, Halfter U, Wang P, Zhu JK. 2005. Role of an Arabidopsis AP2/EREBP-type transcriptional repressor in abscisic acid and drought stress responses. The Plant Cell 17, 2384-2396.

Stockinger EJ, Mao Y, Regier MK, Triezenberg SJ, Thomashow MF. 2001. Transcriptional adaptor and histone acetyltransferase proteins in Arabidopsis and their interactions with CBF1, a transcriptional activator involved in cold-regulated gene expression. Nucleic Acids Research 29, 1524-1533.

Taunton J, Hassig CA, Schreiber SL. 1996. A mammalian histone deacetylase related to a yeast transcriptional regulator Rpd3. Science 272, 408-411.

Tian L, Chen ZJ. 2001. Blocking histone deacetylation in Arabidopsis induces pleiotropic effects on plant gene regulation and development. Proceedings of the National Academy of Sciences, USA 98, 200-205.

Tian L, Wang J, Fong MP, Chen M, Cao H, Gelvin SB, Chen ZJ. 2003. Genetic control of developmental changes induced by disruption of Arabidopsis histone deacetylase 1 (AtHD1) expression. Genetics 165, 399-409.

Vierstra RD. 2003. The ubiquitin/26S proteasome pathway, the complex last chapter in the life of many plant proteins. Trends in Plant Science 8, 135-142.

Weigel D, Glazebrook J. 2002. Arabidopsis: a laboratory manual. Cold Spring Harbor, NY: Cold Spring Harbor Laboratory Press.

Woo HR, Goh CH, Park JH, Teyssendier de la Serve B, Kim JH, Park YI, Nam HG. 2002. Extended leaf longevity in the ore4-1 mutant of Arabidopsis with a reduced expression of a plastid ribosomal protein gene. The Plant Journal 31, 331-340.

Wu K, Malik K, Tian L, Brown D, Miki B. 2000. Functional analysis of a RPD3 histone deacetylase homolog in Arabidopsis thaliana. Plant Molecular Biology 44, 167-176.

Xu C, Wang Y, Li L, Chen W, Xu Z, Bai S. 2005. Histone acetylation affects expression of cellular pattering genes in the Arabidopsis root epidermis. Proceedings of the National Academy of Sciences, USA 102, 14469-14474.

Zhou C, Labbe H, Sridha S, Wang L, Tian L, LatoszekGreen M, Yang Z, Brown D, Miki B, Wu K. 2004. Expression and function of HD2-type histone deacetylases in Arabidopsis development. The Plant Journal 38, 715-724.

Zhou C, Zhang L, Duan J, Miki B, Wu K. 2005. HDA19 is involved in jasmonic acid and ethylene signaling of pathogenresponse in Arabidopsis. The Plant Cell 17, 1196-1204.

Zupkovitz G, Tischler J, Posch M, et al. 2006. Negative and positive regulation of gene expression by mouse histone deacetylase 1. Molecular and Cellular Biology 26, 7913-7928. 